\title{
Formal Design and Analysis of a Wastewater Treatment Control System based on Petri Net
}

\author{
Seno D. Panjaitan ${ }^{1} \&$ Berlian Sitorus $^{2}$ \\ ${ }^{1}$ Automation and Control Group, Dept. of Electrical Engineering, Faculty of \\ Engineering, Tanjungpura University \\ ${ }^{2}$ Dept. of Chemistry, Faculty of Mathematics and Natural Sciences, Tanjungpura \\ University \\ E-mail: senopanjaitan@untan.ac.id
}

\begin{abstract}
This paper proposes a new control design approach for industrial wastewater treatment where its logic control is verifiable. In this research, a treatment control design in a lab-scale was controlled by a microcontroller circuit. The developed system combined anaerobic digestion, aeration and filtration process. Its logic control algorithm was designed by using Signal Interpreted Petri Net. In the logic verification, six analysis properties were satisfied: conflict free (logical process had no conflict behavior), termination (the process could be terminated from any state), non-contradictory outputs, live (any process state could always be reached from other state), deadlock-free, and reversible (the process could always back to initial condition). In the design evaluation, the average value of transparency metrics was 0.984 close to 1 as the best value. The system performance was evaluated by pollutant removal efficiency. The highest removal efficiencies were obtained when each anaerobic and aeration treatment were performed for three days respectively and followed by filtration. Within this condition, the system obtained average removal efficiency $91.7 \%$ of Chemical Oxygen Demand and $95.4 \%$ of Total Suspended Solids. In terms of electricity consumption, the system needed only $1,857.6$ Watt-hour for a batch treatment process.
\end{abstract}

Keywords: aeration; anaerobic; automation; Petri net; wastewater treatment.

\section{Introduction}

Industrial wastewater might contain dissolved organic compounds that require to be reduced before being discharged to the environment. The organic pollutants are commonly removed by biological treatment as aerobic treatment where aeration is the main process. In wastewater treatment, aeration is a process of bringing air into intimate contact with the water. It consumes large amounts of energy mostly to create aeration by using blower. For instance, industries normally need more than one blower for the aeration where one blower usually consumes electrical power about $100 \mathrm{~kW}$ to actuate the motor as the main part of the blower.

Received April $6^{\text {th }}, 2011$, Revised July $15^{\text {th }}, 2011$, Accepted for publication July $25^{\text {h }}, 2011$.

Copyright @ 2012 Published by LPPM ITB \& PII, ISSN: 1978-3051, DOI: 10.5614/itbj.eng.sci.2012.44.1.1 
Another treatment process is anaerobic digestion. It is a simple and effective biotechnological process for minimizing organic wastes from wastewater and generating methane as a beneficial product for the energy source [1]. Anaerobic digestion is the only technology that has proven capable of extracting this energy from wastewaters on a commercial scale [2]. The design concepts of anaerobic treatment systems had been improved from classic reactors like septic tanks and anaerobic ponds, to modern high rate reactor configurations as anaerobic filters, Upward-flow Anaerobic Sludge Blanket, Expanded Granular Sludge Bed, fixed film fluidized bed and expanded bed reactors, and others [3]. According to [2], expensive technology and sophisticated control systems for anaerobic wastewater treatment usually fail at short notice in the developing countries.

In developing world, where capital and skilled labor are not readily available, the preference wastewater treatment should be low-cost technology orientation [4]. Each wastewater treatment needs a control strategy and technology that are appropriate by means of technical acceptability, economic feasibility and social attractiveness. The efforts to get effective designs, which are simple and nonsophisticated equipment, low capital investment costs and low operating and maintenance costs have resulted into the so-called low investment sewage treatment concept [5]. The overall capital and operational expenditure costs should not exceed $30 €$ per inhabitant equivalent per year [6].

This paper presents a new control design approach of automatic wastewater treatment system in a lab scale combining anaerobic digestion, aeration, and filtration. The proposed system consisted of a simple assemblage of inexpensive vessels. The system was used for treating tofu wastewater, a type of food industrial wastewater with high organic pollutants. The system performance was evaluated by means of organic matter and suspended solids removal efficiencies.

\section{Current Control Methods for Wastewater Treatment}

The earlier wastewater treatment control methods were typically dominated by conventional feedback control [7] and optimal control (i.e. linear quadratic) [8], those needed sophisticated controller. The methods were developed for modeling, analysis, and control purposes.

Sequencing Batch Reactor (SBR) was a controlled wastewater treatment reactor that mostly adopted in recent aerobic treatment. SBR was implemented in [9] to treat swine wastewater which is classified as high-strength wastewater. In the treatment, automatic control of carbon addition from an external source was performed in the anoxic and oxic phase. The average removal efficiencies 
achieved for Total Organic Carbon and Nitrogen were 94\% and 96\% respectively. Two bench-scales of SBR using a suitable Carbon/Nitrogen $(\mathrm{C} / \mathrm{N})$ ratio in the influent by adding swine slurry had been developed [10]. As the result, a high total nitrogen removal of $95.5 \%$ was reached.

Another treatment control namely fuzzy logic control was applied by using two parameters: dissolved oxygen and its differential values [11,12]. For this purpose, a fuzzy inference system was constructed with the operator's assistance, while the sensor data was needed to determine the threshold and the influent. Programmable Logic Controller (PLC) was implemented especially for aerobic and anoxic stages. The diagnostic information was inferred by fuzzy logic based on expert knowledge. This system merely played a small role and should be developed in a large scale in order to operate and maintain the system economically.

Another control method for wastewater treatment was predictive control. In [13], a model of predictive control for a wastewater treatment process had simulated. The goal was to have substrate concentration within the standard limits $(<20 \mathrm{mg} / \mathrm{l})$. This goal was achieved by controlling the concentration of dissolved oxygen to a certain value. The controller used neural network as internal model of the process and altered the dilution rate in order to fulfill the control objective. Some problems remained in the implementation such as the need of high computation processor, and certainly the operator should have mathematical background to be able to understand the computation theory.

The previous mentioned approaches were mostly focused on aerobic treatment in order to have optimal total organic Carbon and/or Nitrogen removal by optimizing the aeration process related to the measured dissolved oxygen. The research presented in this paper proposes a combination of anaerobic and aeration processes to achieve optimal removal for Chemical Oxygen Demand (COD) and Total Suspended Solids (TSS). This removal has to be performed to satisfy the water being safe for the environment. The designed combination was also targeted to minimize energy consumption for the optimal treatment.

A more relevant work in terms of control model is presented in [14] where the conventional Petri Net method is adopted to build a simulation tool for online diagnostic on Formosa wastewater treatment plant. However, in terms of modeling, logical model verification and design evaluation was not dealt yet. Signal Interpreted Petri Net (SIPN) presented in the next section as extension of the conventional Petri Net focuses on the logic control and deals with the logical model verification and design evaluation. 
Beside the control method, control hardware is also a concern in the system development. Microcontroller is an inexpensive programmable controller compare to the ones that were applied in previous proposed treatment control, i.e. Personal Computer $[9,10,12]$ and PLC [11]. Using microcontroller in wastewater treatment gave more advantage in terms of cost and mobility, as the treatment needs no high flexibility regarding the applied control strategy. Therefore, the use of microcontroller and its supported circuit are economically feasible for cost-effective industrial wastewater treatment.

\section{Design Approach}

Figure 1 shows the control system diagram of the designed system. Informal specifications represented the control design requirements without considering a formal or theoretical design method. Formalization was performed by using a formal method named SIPN. The SIPN model was then verified to see whether the logic control had satisfied analysis properties. Later on, implementation of control algorithm via microcontroller was carried out. The effluent of the treatment process was then analyzed.

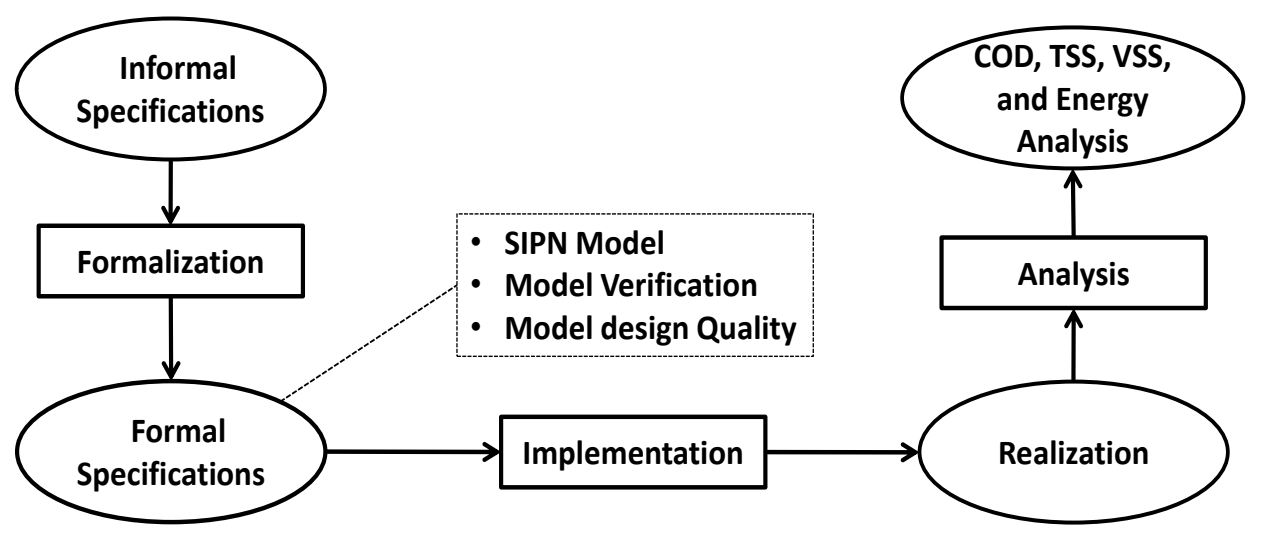

Figure 1 Schematic diagram of wastewater treatment system design.

\subsection{Informal Specifications}

The physical model of a lab-scale wastewater treatment system developed in this research can be seen in Figure 2. The controller consisted of a low-cost microcontroller circuit, signal conditioning, and rectifier. It was connected to two level sensors (i.e. L1, L2), two push buttons (i.e. Start, Stop), and four actuators (i.e. P1, P2, P3, and M1). The control logic was implemented as source code in the microcontroller by utilizing the information of the sensor(s) and actualizing the actuator(s). 
Informal Specifications of the required control logic is as follows (cf. Figure 2):

Initially, a batch of wastewater was transferred to tank T1 from raw industrial wastewater by turning on pump P1.

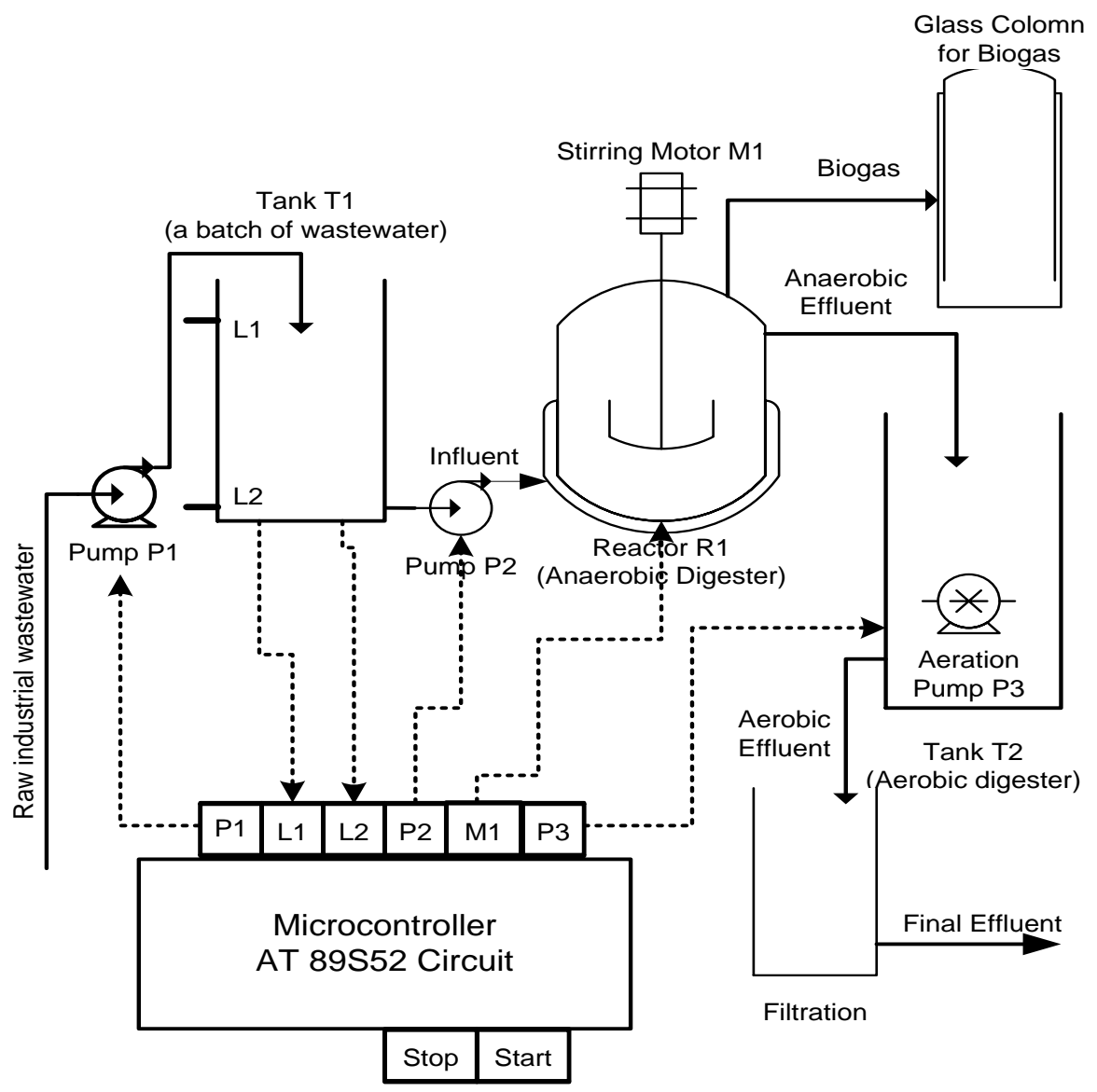

Figure 2 The developed wastewater treatment control: a lab-scale system.

The wastewater was then stored in a temporary tank $\mathrm{T} 1$ before the influent entered the anaerobic digester. The feed control was based on the level sensors which were assembled at tank T1. If the level in tank T1 reached L1 (top level), then pump P1 would be turned off and pump P2 would be turned on afterward.

A batch of water (13 L) was then transferred into anaerobic digester (R1) until the level sensor L2 was off (i.e. level in tank T1 under sensor L2). 
The anaerobic digester was equipped with a stirrer driven by low-speed DC motor. This stirrer was used to give a better contact between the wastewater and cultured bacteria in the anaerobic part. The activation of the stirrer and pumps was controlled by a microcontroller circuit. The stirring motor (M1) was assembled to the reactor R1 and controlled according to a cycle of the timing set per hour, which are 'on' in 1 minute and then 'off' in 59 minutes. This cycle was determined after the previous experiment regarding the optimal timing set for one cycle in anaerobic treatment.

The cycle number for one batch process was depended on the optimum treatment time per batch of process.

The next batch process was started once the previous batch was done. The effluent from R1 moved to tank T2 for aeration.

In the aeration process, a circulation pump (P3) was activated. After being treated for certain of time, the water was brought to filtration compartment to get the final effluent.

\subsection{Formal Specifications}

\subsubsection{Modeling of Logic Control based on SIPN}

SIPN method adopted from [15] was taken into account for modeling the control logic behavior of the treatment process. SIPN consists of nine-tuple as $S I P N=\left(P, T, F, \mathbf{m}_{0}, I, O, \varphi, \varpi, \Omega\right)$ where,

$\boldsymbol{P} \quad: \mathrm{P}=\left\{p_{1}, \ldots, p_{|P|}\right\}$, a limited set of places, denoted as circles,

$\boldsymbol{T}: \mathrm{T}=\left\{t_{l}, \ldots, t_{|T|}\right\}$, a limited set of transitions, denoted as bars,

$\boldsymbol{F} \quad: \quad \mathrm{F} \subseteq \boldsymbol{P} \times \boldsymbol{T} \cup \boldsymbol{T} \times \boldsymbol{P}$, a limited and non-empty set arcs which

connect place to transition or vice versa, but connection from place to place or transition to transition is not allowed, denoted as arrows,

I : a limited and non-empty set of signal inputs,

$\boldsymbol{O} \quad$ : a limited and non-empty set of output signals with $I \cap O=\varnothing$,

$\varphi \quad$ : a mapping that associate any transition to a firing condition,

$\boldsymbol{\varpi} \quad$ : a mapping that associates any place to an output: $\mathrm{p} \rightarrow\{0,1,-\}^{|0|}$, 
$\Omega \quad: \quad \mathbf{m} \rightarrow\{-, 1,0, c\}^{|O|}$ a output function of a SIPN model.

List of inputs and outputs are listed in Table 1 and 2 prior to the modeling. Timers in the microcontroller were applied for delaying a process.

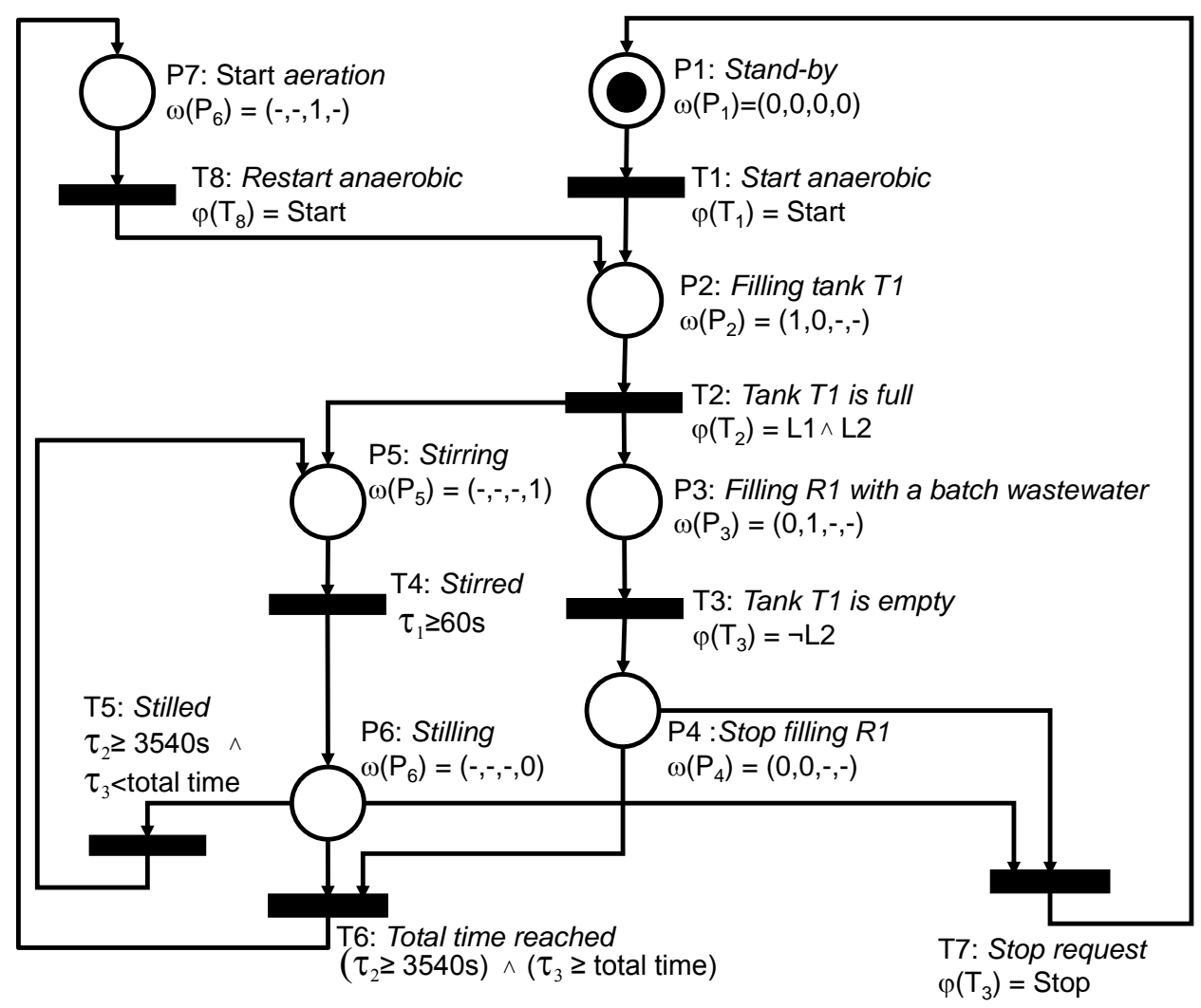

Figure 3 SIPN model of the treatment control logic design.

The treatment control logic designed by SIPN model is shown in Figure 3 using inputs and outputs in Table 1 and Table 2 . The model had seven places, $P=$ ( $p 1, p 2, p 3, p 4, p 5, p 6, p 7)$, eight transitions, $T=(t 1, t 2, t 3, t 4, t 5, t 6, t 7, t 8)$ and nineteen arcs, $F=\{(p 1, t 1),(t 1, p 2),(p 2, t 2),(t 2, p 3),(p 3, t 3),(t 3, p 4),(t 2, p 5)$, $(p 5, t 4),(t 4, p 6), \quad(p 4, t 6), \quad(p 6, t 5),(t 5, p 5),(p 6, t 6), \quad(p 4, t 7),(p 6, t 7),(t 7, p 1)$, $(t 6, p 7),(p 7, t 8),(t 8, p 2)\}$. The number of place, transition, and arc shows the size of model therefore the smaller size model the better. The developed model had a small size. 
Table 1 List of the System Inputs (I).

\begin{tabular}{cl}
\hline $\mathbf{I}$ & \multicolumn{1}{c}{ Logical Value "1" } \\
\hline$i_{1}$ & $\begin{array}{l}\text { Start button is pressed to start or restart the anaerobic } \\
\text { digestion }\end{array}$ \\
$i_{2}$ & $\begin{array}{l}\text { Stop button is pressed to stop the current process } \\
i_{3}\end{array}$ \\
$i_{4}$ & Top level sensor (L1) of Temporary tank T1 is reached \\
& Bottom level sensor (L2) of Temporary tank T1 is \\
& reached
\end{tabular}

Table 2 List of the System Outputs $(O)$.

\begin{tabular}{cl}
\hline O & \multicolumn{1}{c}{ Logical Value "1" } \\
\hline$o_{1}$ & Pump P1 is turned on to transfer wastewater to tank T1 \\
$o_{2}$ & Peristaltic Pump P2 is turned on to transfer a batch (13 L) \\
& wastewater to reactor R1 (anaerobic digester) \\
$o_{3}$ & Circulation pump P3 is turned on for aeration \\
$o_{4}$ & Stirring motor (M1) for anaerobic process is turned on \\
\hline
\end{tabular}

Outputs in place $\mathrm{P}_{\mathrm{n}}$ were represented as the value of $\omega\left(\mathrm{P}_{\mathrm{n}}\right)$ where $n$ is the place number. Since the model had four actuators or outputs (see Table 2) then the outputs value was represented $\omega\left(\mathrm{P}_{\mathrm{n}}\right)=\left(\mathrm{o}_{1}, \mathrm{O}_{2}, \mathrm{O}_{3}, \mathrm{O}_{4}\right)$. The value of each output could be 0 ("low"), 1 ("high"), or - ("don't care"). On the other side, the logical condition that had to be fulfilled in transition $\mathrm{T}_{\mathrm{m}}$ was denoted as $\varphi\left(\mathrm{T}_{\mathrm{m}}\right)$, where $m$ represents the transition number.

A token shows the dynamical process represented by SIPN. In the initial condition, a token was put in place P1. It represented that the current state was initially in place $P 1$ (i.e. Stand-by state) and the four outputs (see Table 2) were " 0 " or off. The notation of $(0,0,0,0)$ in $P 1$ stated that $\mathrm{o}_{1}:=" 0 ", \mathrm{o}_{2}:=" 0 ", \mathrm{o}_{3}:=$ "0", and $0_{4}:=" 0 "$ respectively. Thus, if the Start button was pressed then transition $T 1$ was enabled and it allowed the token moved from $P 1$ to $P 2$. This meant the state of the process moved from place $P 1$ to $P 2$ (i.e. Filling tank 1 state).

The outputs value was updated to $\omega\left(\mathrm{P}_{2}\right)=(1,0,-,-)$ which meant Pump1 was turned on $(\mathrm{o} 1:=1)$ and Pump2 was turned off $\left(\mathrm{o}_{2}:=0\right)$, while Pump3 $\left(\mathrm{o}_{3}\right)$ and M1 $\left(\mathrm{o}_{4}\right)$ were "don't care". If tank $\mathrm{T} 1$ was full when the condition " $\mathrm{i}_{3} \Lambda \mathrm{i}_{4}$ " was true ( $\mathrm{i}_{3}$ and $\mathrm{i}_{4}$ were " 1 "), transition $T 2$ was enabled. As the result, a token moved from place P2 to P3 (i.e. stirring the reactor) and P5 (i.e. filling anaerobic reactor with a batch of wastewater) which ran concurrently. After $60 \mathrm{~s}$ stirring water in the reactor, a token moved from place P5 to P6 (i.e. Still state) by 
turning off the $\mathrm{o}_{4}$, i.e. $\omega\left(\mathrm{P}_{6}\right)=(-,-,-, 0)$. If the process was not reached the determined total time yet while still time was reached, then the process returned to place P5 for the next cycle until the determined total time of one batch process was achieved.

On the other side, once the tank $\mathrm{T} 1$ was empty after the filling process of reactor R1 (i.e. place P3) then the token of process moved to place P4. If there was a token in each place $P 4$ and $P 6$ (i.e. synchronization between filling and stirring process), then transition $T 6$ could be enabled. Once this transition enabled, the tokens in the places were left and one token moved to place $P 7$ to activate the aeration. Afterwards, the anaerobic process was restarted directly from place P2 for the next batch while the aeration process runs concurrently. The processes in place P4 and P6 could be stopped by enabling the transition $T 7$ and then returned to initial state (i.e. place $P 1$ ).

\subsubsection{Verification the Developed SIPN Model}

Verification was performed to analyze whether the formal specification or the control logic in the SIPN model was correctly built. It included five analysis properties, i.e. conflict freeness, termination, formally correct output, deadlock freeness, and reversibility. A reachability graph of the SIPN $\left(\mathrm{RG}_{\mathrm{SIPN}}\right)$ has to be generated for this purpose. For SIPN model in Figure 3, its $\mathrm{RG}_{\text {SIPN }}$ is shown in Figure 4. The $\mathrm{RG}_{\text {SIPN }}$ had seven states related to the behavior of markings (dynamical model of token movement) including the related representation of the outputs (i.e. $\Omega$ ). Since the SIPN model had seven places, then the marking had also seven elements. For instance, marking in the initial state (i.e. place P1) was $m_{0}=(1,0,0,0,0,0,0)$, which meant in this state there was a token only in $P 2$, while no token in place P2, P3, P4, P5, P6, and P7. For the second state $\left(m_{2}=\right.$ $(0,0,1,0,1,0,0)$, there were a token in each place $P 3$ and $P 5$ (i.e. concurrent process) while other places had no token.

For conflict-freeness property, algorithm is deterministic if and only if the firing conditions at every branching in $\mathrm{RG}_{\text {SIPN }}$ are disjoint. In the $\mathrm{RG}_{\text {SIPN }}$, it seemed that there was a conflict in case $m_{2}$ when transition $T 3$ and $T 4$ were enabled in the same time. However, in Figure 3, these transitions had guard conditions which could not be enabled simultaneously and independent preplace (input place to a particular transition). Therefore, the developed SIPN model was conflict-free.

Termination is a property to verify whether the algorithm can be terminated. The algorithm terminates if and only if there is no self-loop at any state in $\mathrm{RG}_{\mathrm{SIPN}}$. It could not found any self-loop in the $\mathrm{RG}_{\mathrm{SIPN}}$, therefore the developed SIPN model fulfilled the termination property. 


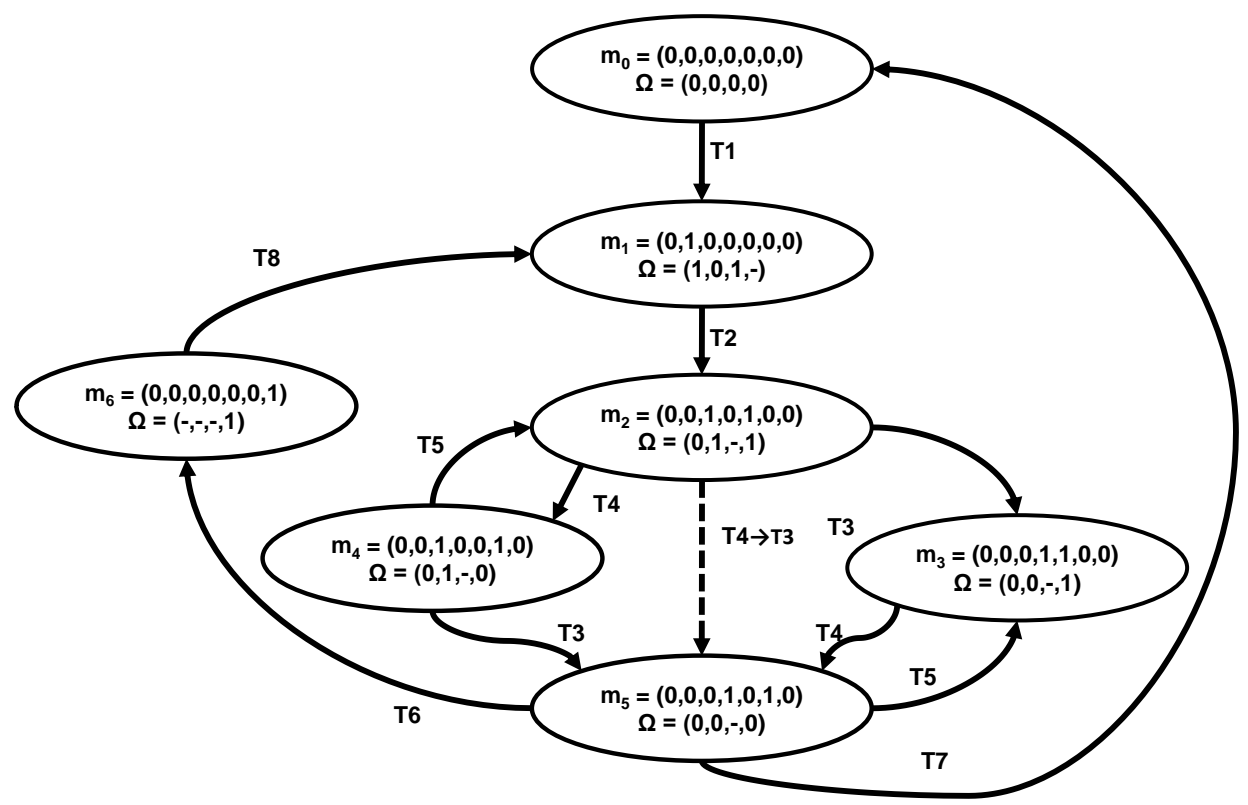

Figure $4 \mathrm{RG}_{\mathrm{SIPN}}$ of the Treatment Control.

The output of an SIPN is formally correct if and only if it is specified and noncontradictory: $\Omega_{\mathrm{N}}(\mathrm{SIPN}) \in\{1,0, \mathrm{~d}\}^{|\mathrm{O}|}$. The $\Omega_{\mathrm{N}}(\mathrm{SIPN})$ of the model was $(\mathrm{d}, \mathrm{d}, \mathrm{o}, \mathrm{o})$. The first and the second outputs (Pump1 and Pump2 respectively) were " $\mathrm{d}$ " (defined) which means that the signals were always defined either 1 or 0 in the SIPN. The third and fourth outputs were "o" (other possible combination and non-contradictory) since its value varied on "1", " 0 ", or "-". The developed SIPN model had specified and non-contradictory outputs.

For liveness property, there has to be a path from every state in $\mathrm{RG}_{\text {SIPN }}$ containing all transition. The $\mathrm{RG}_{\mathrm{SIPN}}$ in Figure 4 shows that the built SIPN was live. Deadlock-freeness property is fulfilled if there is no state in $\mathrm{RG}_{\text {SIPN }}$ without an outgoing arc. The built SIPN was then deadlock-free. The last property is reversibility. Reversibility property is satisfied if and only if from every marking in $\mathrm{RG}_{\text {SIPN }}$ a path back to $m_{0}$ exists. The built SIPN was reversible since the process could back to initial state via transition T7.

\subsubsection{Evaluation of the Design Quality}

There were some evaluation criteria [15] of the programming code or software. Ten evaluation criteria were used in this research in order to evaluate the quality of the built SIPN model. The transparency metric value of the quality was in the range 0 (the worst) to 1 (the best). The computation summary of the SIPN model evaluation based on transparency metrics is listed in Table 3. 
Table 3 Transparency metrics of the logic control design.

\begin{tabular}{|c|c|c|}
\hline Criterion & Formula & Metric value \\
\hline Comments (E1) & $\frac{\text { \#comments }}{\text { \#places }+\# \text { transitims }}$ & $E 1=\frac{13}{6+7}=1$ \\
\hline $\begin{array}{l}\text { Directionality } \\
\text { (E2) }\end{array}$ & $\frac{\# \text { Arcsin preferred direction }}{\# \text { arcs }}$ & $E 2=\frac{19}{19}=1$ \\
\hline $\begin{array}{l}\text { No Intersection } \\
\text { (E3) }\end{array}$ & $1-\frac{\# \text { Intersected } \arcsin \text { SIPN }}{\# \text { arcs in SIPN }}$ & $E 3=1-\frac{2}{19}=0.89$ \\
\hline $\begin{array}{l}\text { No redundant } \\
\text { input signals } \\
\text { (E4) }\end{array}$ & $1-\frac{\text { \#Redundantinputsignals }}{\text { \#inputsignals }}$ & $E 4=1-\frac{0}{4}=1$ \\
\hline $\begin{array}{l}\text { No redundant } \\
\text { output signals } \\
\text { (E5) }\end{array}$ & $1-\frac{\text { \#Redundantoutputsignals }}{\text { \#ouputsignals }}$ & $E 5=1-\frac{0}{4}=1$ \\
\hline $\begin{array}{l}\text { No redundant } \\
\text { output settings } \\
\text { (E6) }\end{array}$ & $\frac{\text { \#Formallycorrect non- redundantoutputsettingin } \mathrm{RG}_{\text {SIPN }}}{\max (1, \text { \#outputassignmentin SIPNmarkings })}$ & $E 5=1$ \\
\hline Safety (E7) & $1-\frac{\# \text { Unsafestatesin } \mathrm{aRG}_{\mathrm{SIPN}}}{\# \text { Statesin } \mathrm{aRG}_{\mathrm{SIPN}}}$ & $E 7=1-\frac{0}{5}=1$ \\
\hline $\begin{array}{c}\text { No dynamic } \\
\text { synchronization } \\
\text { (E8) }\end{array}$ & $1-\frac{\# \text { Arcs due to DSin } R_{\text {SIPN }}}{\max \left(1, \# \arcsin R_{\text {SIPN }}\right)}$ & $E 8=1-\frac{0}{19}=1$ \\
\hline $\begin{array}{l}\text { Low expression } \\
\text { complexity } \\
\text { (E9) }\end{array}$ & $1-\frac{\max \left(\min \left(E C_{S I P N}, 5\right), 3\right)-3}{2}$ & $E 9=1-\frac{0}{2}=1$ \\
\hline $\begin{array}{l}\text { Low Net } \\
\text { Complexity } \\
\text { (E10) }\end{array}$ & $1-\frac{\max (\min (N C, 100), 25)-25}{75}$ & $E 10=1-\frac{3.66}{75}=0.95$ \\
\hline $\begin{array}{c}\text { Overall } \\
\text { transparency } \\
\text { metric value } \\
\text { (T) }\end{array}$ & $T=\frac{\sum_{i=1}^{10} c_{i} \cdot t_{i}}{\sum_{i=1}^{10} c_{i}}$ & $T=\frac{9.84}{10}=0.984$ \\
\hline
\end{tabular}

The first criterion was comments (E1). The SIPN was easier understood when the more comments were used in a SIPN model. The graphical representation of the model was comprehensible if exists a preferred direction, i.e directionality (E2) criterion. The direction must be determined by the designer. In our research, top-down was the preferred direction where the arc points downwards 
when the arrowhead points downward. A further graphical criterion was nointersections (E3) which could hinders understanding is the existence of intersecting arcs in the SIPN model.

Redundant information may be found at different elements in an SIPN. There were three criteria for the redundancies, i.e. no redundant input signals, no redundant output signals, and no redundant output settings. If an input signal has no influence on the SIPN it was said to be redundant. No redundant input signals (E4) criterion provided the availability of this redundant signals information. Furthermore, an output signal was called redundant if it is set constantly in the same value which is the case of misleading redundant information. No redundant output signals (E5) criterion provided the availability of these redundant signals. No redundant output settings (E7) evaluates that outputs should only be affected by one place in each marking. The number of non-redundant output settings is set in relation to the number of output assignments in the SIPN markings.

The next type of the evaluation is visible dynamics which compounds two criteria, i.e. safety and no dynamic synchronization. These criteria were evaluated on the augmented $\mathrm{RG}_{\mathrm{SIPN}}\left(\mathrm{aRG}_{\mathrm{SIPN}}\right)$. However, in the built system, the $\mathrm{aRG}_{\text {SIPN }}$ was the same with the generated $\mathrm{RG}_{\text {SIPN. }}$. An SIPN is safe (E7) if only if there was no node or state in $\mathrm{aRG}_{\text {SIPN }}$ with a component in the marking value greater than one. Dynamic synchronization (DS) is the effect in SIPN when several transitions fire simultaneously. No dynamic synchronization (E8) criterion provided this the effect.

Complexity had two criteria, i.e. low expression complexity (E9) and low net complexity (E10). Expression Complexity $(E C)$ of an SIPN is the ratio of the expression complexity ec $\left(\mathrm{t}_{\mathrm{i}}\right)$ of all its transition to the number of transitions such as input signal $(\mathrm{EC}=0)$, Not $(\mathrm{EC}=1)$, And/Or $(\mathrm{EC}=2)$, and NAND/NOR $(\mathrm{EC}=3)$. The $\mathbf{E 9}$ value of the built SIPN needed the value of EC. $\mathrm{EC}$ of the built SIPN was as follows:

$$
\begin{aligned}
E C_{S I P N}=\frac{\sum_{\mathrm{t}=1}^{|\mathrm{T}|} \mathrm{ec}\left(\mathrm{t}_{1}\right)}{\# \text { transitions }}= & \frac{\mathrm{ec}\left(\mathrm{t}_{1}\right)+\mathrm{ec}\left(\mathrm{t}_{2}\right)+\mathrm{ec}\left(\mathrm{t}_{3}\right)+\mathrm{ec}\left(\mathrm{t}_{4}\right)+\mathrm{ec}\left(\mathrm{t}_{5}\right)+\mathrm{ec}\left(\mathrm{t}_{6}\right)+\mathrm{ec}\left(\mathrm{t}_{7}\right)+\mathrm{ec}\left(\mathrm{t}_{8}\right)}{8} \\
& =\frac{0+2+1+0+2+2+0+0}{8}=\frac{7}{8}
\end{aligned}
$$

The last evaluation criterion was low net complexity (E10). To have the value of E10, the value of net complexity (NC) was computed. The measure NC if composed of Structural Complexity of SIPN ( $\left.\mathrm{SC}_{\mathrm{SIPN}}\right)$, Hierarchical Complexity of SIPN ( $\left.\mathrm{HC}_{\mathrm{SIPN}}\right)$, Unstructuredness of SIPNI $\left(\mathrm{MCC}_{\mathrm{SIPN}}\right)$, and Branching of 
SIPN $\left(\mathrm{B}_{\text {SIPN }}\right) . \mathrm{SC}_{\text {SIPN }}$ rates the complexity of the SIPN structured. It relates the number of arcs in a Petri Net to the number of modules. The $\mathrm{SC}_{\text {SIPN }}$ of the built SIPN is as follows,

$$
\mathrm{SC}_{\mathrm{SIPN}}=\frac{|\mathrm{F}|}{\mathrm{M}}=\frac{19}{1}=19
$$

where: $|\mathrm{F}|=$ number of arcs in the SIPN, $\mathrm{M}=$ number of modules in the SIPN.

The measure of $\mathrm{HC}_{\text {SIPN }}$ relates the number of modules in an SIPN to the depth of the hierarchical structured or number of levels. The value $\mathrm{HC}_{\mathrm{SIPN}}$ of the developed SIPN was as follows,

$$
\mathrm{HC}_{\mathrm{SIPN}}=\frac{\mathrm{M}}{\mathrm{L}}=\frac{1}{1}=1
$$

where: $\mathrm{L}=$ number of levels in the SIPN.

$\mathrm{MCC}_{\text {SIPN }}$ evaluates the number of modules (M), cycles (C), and decision places $\left(\left|\mathrm{D}_{\mathrm{P}}\right|\right)$. The built SIPN contained two decision places (i.e. place P4, P6), therefore $\left|D_{P}\right|=2$ holds. There was only one module $(M=1)$. A cycle in an SIPN is a sequence of arcs. Two cycles are equal if they contain the same arcs. The built SIPN owned four cycles $(\mathrm{C}=4)$, i.e. $(p 1, t 1, p 2, t 2, p 3, t 3, p 4, t 7, p 1),(p 1$, $t 1, p 2, t 2, p 5, p 6, t 7, p 1),(p 2, t 2, p 5, t 4, p 6, t 6, p 7, t 7, p 2)$, and $(p 5, t 4, p 6, t 5$, $p 5)$. Then the $\mathrm{MCC}_{\text {SIPN }}$ was,

$$
\mathrm{MCC}_{\mathrm{SIPN}}=\left|\mathrm{D}_{\mathrm{P}}\right|-\mathrm{M}+\mathrm{C}+1=2-1+4+1=6
$$

The measure Branching $\left(\mathrm{B}_{\text {SIPN }}\right)$ relates the weighted sum of decision vertices of all subgraphs of a $R_{\text {SIPN }}$ to the weighted sum of decision vertices in the RG. If there are no decision vertices, then $\mathrm{B}_{\text {SIPN }}$ is zero. The definition of Branching is as follows,

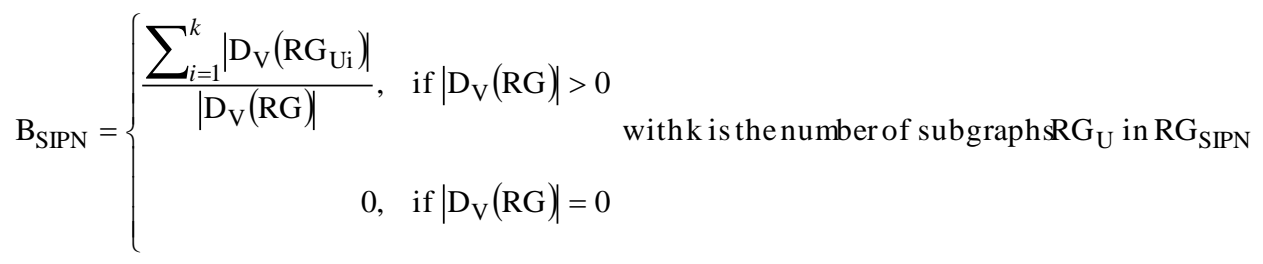

The built $\mathrm{RG}_{\text {SIPN }}$ (see Figure 4) contains three decision vertices $\left(\mathbf{m}_{\mathbf{2}}, \mathbf{m}_{\mathbf{4}}\right.$, and $\left.\mathbf{m}_{\mathbf{5}}\right)$. These three vertices composes eight subgraphs $\left(\mathrm{RG}_{\mathrm{U}}\right)$, therefore $\mathrm{B}_{\mathrm{SIPN}}$ is equal to $2.66\left(\mathrm{~B}_{\mathrm{SIPN}}=8 / 3=2.66\right)$. 
The combined complexity measure Net Complexity $(N C)$ is the sum of the four measures presented above,

$$
\begin{aligned}
\mathrm{NC} & =\mathrm{SC}_{\mathrm{SIPN}}+\mathrm{HC}_{\mathrm{SIPN}}+\mathrm{MCC}_{\mathrm{SIPN}}+\mathrm{B}_{\mathrm{SIPN}} \\
& =19+1+6+2.66=28.66
\end{aligned}
$$

The NC value was used to compute E10) in Table 3.

The overall transparency metric of the built model was 0.984 (see Table 3) which was close to 1 as the best value.

\subsection{Realization of the Design}

The built SIPN was realized by using a programming code (i.e. Assembly language) that was uploaded in a microcontroller and being applied on the wastewater treatment control afterward. By using the SIPN, the process could be verified and evaluated easily and the code could be reprogrammed immediately. The physical model of the controller circuit and their controlled plant can be seen in Figure 2.

\section{$4 \quad$ Wastewater System Performance Analysis}

The performance of wastewater treatment system was analyzed using two parameters: COD and TSS. The COD concentration related to amount of organic compounds in the water body, while TSS indicates the amount of suspended solids deposits in the water. A high concentration of COD parameter leads to the depletion of natural oxygen resources and development of septic condition in aquatic environment, which could make severe condition if wastewater discharged untreated to water bodies.

The untreated wastewater used in this research had a high concentration regarding the two parameters, which were $\pm 22080 \mathrm{mg} / \mathrm{l}$ and $1576 \mathrm{mg} / \mathrm{l}$ for COD and TSS respectively. The controller used for an automatic system was first operated in different hydraulic retention times in anaerobic part. Treating in four different times (1-4 days) was meant to investigate the optimum time needed for wastewater to be treated in the anaerobic process. The experimental result of the wastewater system during the anaerobic process is presented in Figure 5. This graph consisted of one $x$-axis and two $y$-axes. The $x$-axis indicates Hydraulic Retention Time or treatment time needed in anaerobic process, whereas the $y$-axes are referred to COD and TSS concentrations of water that being analyzed. Thus, the concentrations of COD and TSS in 0 day are the initial concentrations in raw wastewater, while in 1 through 4 demonstrate the results analyzed during anaerobic process for both parameters. 
Figure 5 describes that the lowest concentrations for both COD and TSS of the water effluent were obtained during three-day treatment, which were \pm 3536 $\mathrm{mg} / \mathrm{l}$ for COD and $248 \mathrm{mg} / \mathrm{l}$ for TSS. By comparing pollutant parameters concentrations in the raw wastewater and the effluent water after anaerobic process, it was concluded that the anaerobic process was able to remove $\pm 84 \%$ pollutants from the water in terms of COD and $84.3 \%$ concerning TSS. Consequently, during the treatment throughout this research, three-day treatment was used as the optimum retention for the anaerobic process, which was followed by aeration process afterward. The decreasing in COD concentration within wastewater treated was due to the organic compounds was degraded by anaerobic bacteria in the anaerobic digester. According to [16], organic compounds in wastewater could be classified as soluble and suspended organic compounds. The suspended compounds could be hydrolyzed by bacteria and converted into simpler compounds that later were subjected into acidification. The soluble compounds directly acidified into acetic, lactic, propionic and butiric acids.

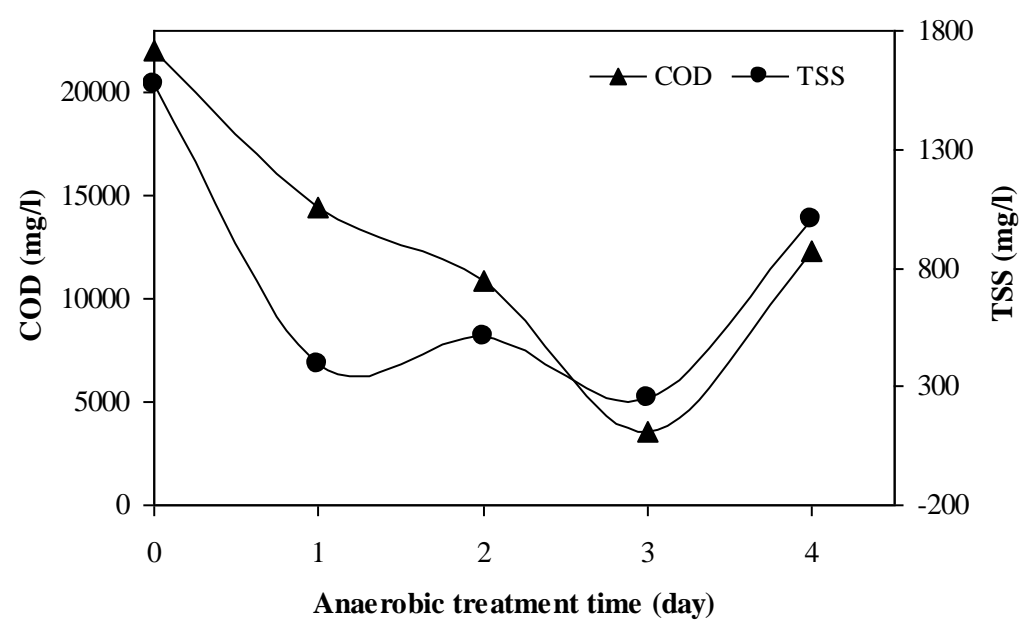

Figure 5 Average concentrations of COD and TSS during anaerobic process.

Figure 6 depicts the average removal efficiencies in the final water effluent after a series of treatment, where (a) refers to results analyses of COD and TSS after aeration treatment and (b) presents the results analyses after aeration followed by filtration. The graphs consist of one $x$-axis and two $y$-axes similar to the previous graph in Figure 5. However, the concentrations of COD and TSS in 0 day are concentrations achieved after 3-day anaerobic treatment in the series of treatment, while 1 through 4 demonstrate the concentrations during anaerobic process. For instance 1-day aeration treatment means that the wastewater has 
been treated four days: three days in anaerobic digestion and 1-day aeration process respectively.

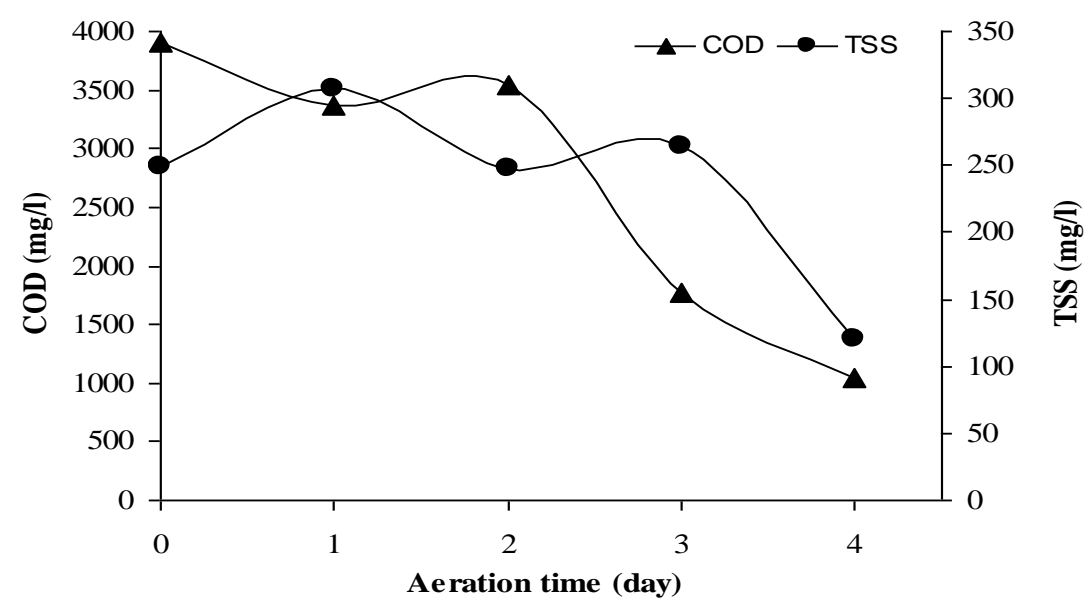

(a)

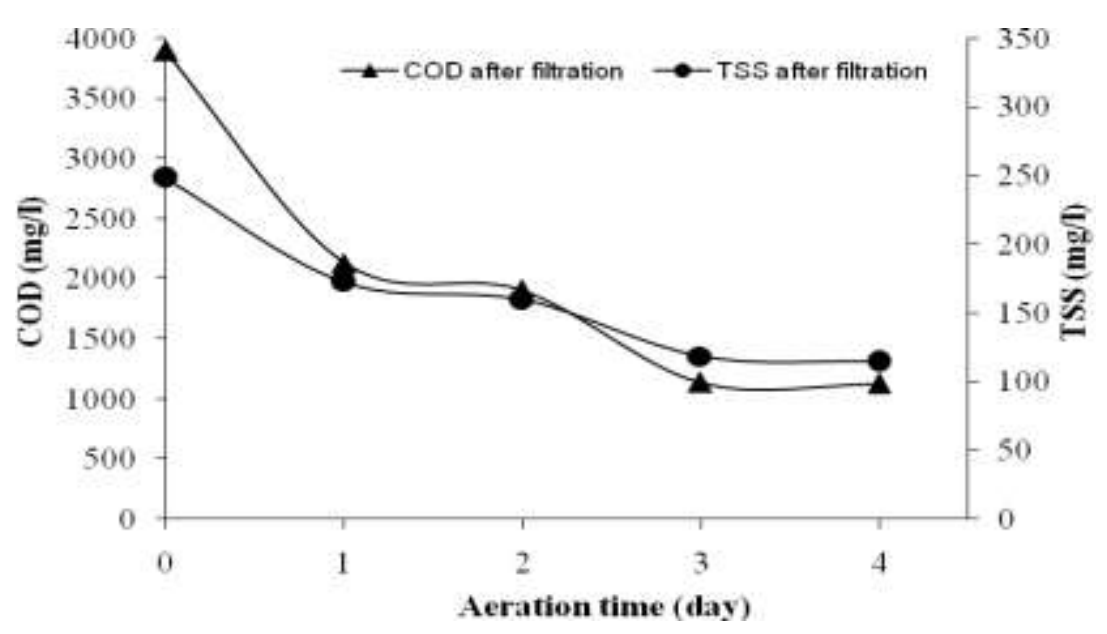

(b)

Figure 6 Average concentrations of COD and TSS after: (a) aeration process; (b) aeration and filtration.

The COD concentrations in (a) reached the lowest concentration within 4-day treatment which was $1165 \mathrm{mg} / \mathrm{l}$, compared to $1768 \mathrm{mg} / \mathrm{l}$ in 3-day-treatment. In (b) the COD concentration in 4-day-treatment was $1128 \mathrm{mg} / \mathrm{l}$ while during 3day aeration it was $1140 \mathrm{mg} / \mathrm{l}$. Concerning TSS concentrations, from (a) it can be seen that the lowest concentrations for TSS was obtained in 4-day-treatment 
which was $120 \mathrm{mg} / \mathrm{l}$ but in with filtration as in (b) the TSS concentrations of 3day was $118 \mathrm{mg} / \mathrm{l}$ and $115 \mathrm{mg} / \mathrm{l}$ in 4-day treatment.

The reduction of pollutants concentration was due to the production of dissolved oxygen (DO) by water recycling in the aeration compartment also gave a contribution to make the process more effective. In addition, aeration diminished the disadvantage of the anaerobic digestion concerning effluent odor problem. Moreover, filtration process using a composition of filter media in the last compartment affected the suspended solids removal efficiency.

Beside the pollutant concentration in the effluent water, the amount of energy/electricity needed for a series of treatment combination was being considered in choosing the preferred type of treatment. The energy consumption in terms of electricity of each batch process of treatment control can be explained as the following. During anaerobic process, electricity was needed for controller, stirring motor and two pumps (P1 and P2), while in the aeration process the electricity was needed for controller and circulation pump (P3). Controller in anaerobic and aeration process needed only $39.6 \mathrm{Wh} / 1$ day, 79.2 $\mathrm{Wh} / 2$ days, or $118.8 \mathrm{Wh} / 3$ days since it worked 24 hours in a day, 48 hours in two days, or 72 hours in three days. Stirring motor needed only $2.592 \mathrm{Wh} /$ day since it worked only 1 minute/hour which meant in 24 hours it worked for 24 minutes. Pump P2 and Pump P1 consumed very low energy since they ran in a short time, i.e. for wastewater transfer. Therefore, for a combination of 3-day anaerobic treatment with 3-day aeration and filtration, the process needed 1,857.6 Watt-hour, while a series treatment that consisted of 3-day-anaerobic treatment with 4-day-aeration and filtration needed electricity of 2,473.20 Watthour.

Besides the energy needed, an amount of energy was also produced by the treatment process which was biogas produced from the anaerobic process. The amount of biogas was expressed as volume of biogas produced per day during the anaerobic treatment. In average $1,580 \mathrm{~mL}$ of biogas was produced per day in the anaerobic treatment.

From a statistical analysis called post-hoc test that was carried out (data not shown), it was reckoned that the COD concentration in 3-day and 4-day without filtration treatment were not significantly different with the COD concentration in 3-day and 4-day treatment with filtration. Moreover, although TSS concentration in 4-day-treatment (a) was the lowest compared to others, yet it was not significantly different with TSS concentration in 3-day and 4-day treatment with filtration. Likewise, treatment condition needed shorter aeration time was the preference since the longer aeration process meant the higher energy needed. 


\section{Conclusions}

This paper has elucidated a new design approach of wastewater treatment control by combining anaerobic, aeration, and filtration process, which were controlled by a low-cost microcontroller circuit as the main processor. The logic control design was modeled by using SIPN. From verification result, the logic design was proven to satisfy six properties: conflict free, termination, noncontradictory outputs, live, deadlock-free, and reversible. From the design evaluation, the average value of transparency metrics was 0.984 close to 1 as the best value. The experiment has been carried out in a lab-scale wastewater treatment system. The preferred treatment condition considered as the optimum waste water treatment time in this research was 3-day-anaerobic combined with 3-day-aeration, and direct filtration. Within this condition, the treatment system needed electricity of $1,857.6$ Watt-hour per a batch of process, and had removal efficiency of $91.7 \%$ for COD and $95.4 \%$ for TSS.

\section{Acknowledgement}

We would like to thank Indonesia Toray Science Foundation (ITSF) for funding the research.

\section{References}

[1] Leitão, R.C., van Haandel, A.C., Zeeman, G. \& Lettinga, G., The Effects of Operational and Environmental Variations on Anaerobic Wastewater Treatment Systems: A Review, Bioresource Technology, 97(9), 11051118, 2006.

[2] Aiyuk, S. E., Forrez, I., De Kempeneer, L., van Haandel, A. \& Verstraete, W., Anaerobic and Complementary Treatment of Domestic Sewage in Regions with Hot Climates: A Review, Bioresource Technology, 97(17), 2225-2241, 2006.

[3] Van Haandel, A., Kato, M. T., Cavalcanti, P. \& Florencio, L., Anaerobic Reactor Design Concepts for the Treatment of Domestic Wastewater, Reviews in Environmental Science and Biotechnology, 5(1), 21-38, 2006.

[4] Sandino, J., Selecting Appropriate Wastewater Treatment Technologies for Large Urban Applications in Developing Countries, Water 21, 1(1), 42-44, 2007.

[5] Sitorus, B. New Development in Low Investment Sewage Treatment, Verlag Dr. Müller, Saarbrücken-Germany, 2010. 
[6] Mendoza, L., Carballa, M., Sitorus, B., Pieters, J. \& Verstraete, W., Technical and Economic Feasibility of Gradual Concentric Chambers Reactor for Sewage Treatment in Developing Countries, Electronic Journal of Biotechnology, 12(2), 1-13, 2009.

[7] Olsson, G. \& Newell, R.B., Wastewater Treatment Systems - Modelling, Diagnosis and Control, IWA Publishing, 1999.

[8] Devisscher, M., Harmand, J., Steyer, J.P. \& Vanrolleghem, P.A., Control Design of An Industrial Equalization System - Handling System Constraints, Actuator Faults and Varying Operating Conditions, Proc. IFAC 4th Symposium on Fault Detection, Supervision and Safety for Technical Processes, IFAC, 2000.

[9] Kim, J., Chenb, M., Kishidac, N. \& Sudoa, R., Integrated Real-Time Control Strategy for Nitrogen Removal in Swine Wastewater Treatment Using Sequencing Batch Reactors, Water Research, 38(14-15), 33403348, 2004.

[10] Kishida, N., Kim, J., Chen, M., Sasaki, H. \& Sudo, R., Effectiveness of Oxidation-Reduction Potential and $p H$ as Monitoring and Control Parameters for Nitrogen Removal in Swine Wastewater Treatment by Sequencing Batch Reactors, Journal of Bioscience and Bioengineering, 96(3), 285-290, 2003.

[11] Bae, H., Kim, S., Lee, M.H., Kim, Y. \& Kim, C.W., Knowledge-based Unmanned Automation and Control Systems for the SBR Wastewater Treatment Process, Artif Life Robotics, 7, 107-111, 2003.

[12] Jun, B., Park, J. \& Chun, M., Knowledge-Based Fuzzy Control of PilotScale SBR for Wastewater Treatment, R. Khosla et al. (Eds.): KES 2005, LNAI 3682, Springer-Verlag Berlin Heidelberg, pp. 608-614, 2005.

[13] Caraman, S., Sbarciog, M. \& Barbu, M., Predictive Control of a Wastewater Treatment Process, International Journal of Computers, Communications \& Control, 2(2), 132-142, 2007.

[14] Yao, A.W.L., Ji, Z. \& Liao, H.T., A Petri Nets-based Process Planning System for Wastewater Treatment, Asian Journal of Control, 12(3), 281291, 2009.

[15] Frey, G., Design and Formal Analysis of Petri Net based Logic Control Algorithms, Shaker Verlag, Aachen, 2002. 
[16] Bravo, A. D., Carballa, M., Filippi, R. G. \& Chamy, R., Treatment of Low Strength Sewage with High Suspended Organic Matter Content in An Anaerobic Sequencing Batch Reactor and Modeling Application, Electronic Journal of Biotechnology, 12(3), 1-10, 2009. 